\title{
K-coverage prediction optimization for non-uniform motion objects in wireless video sensor networks
}

\author{
Yibo Jiang ${ }^{\mathrm{a}}$, Shanghao Sheng, Nianhua Wang and Jiadong Mei \\ College of Computer Science, Zhejiang University of Technology, Hangzhou, China
}

\begin{abstract}
The existing wireless video sensor networks tracking algorithms have much higher performance requirements of the sensor node in networks. For solving this problem, we propose a new target trajectory prediction model for non-uniform motion, which takes all the possible locations of each target at next moment into consideration. Furthermore, we design a K-coverage dynamic optimization algorithm to gain benefit of distributed method based on the aforementioned mathematical model. The experimental results indicate that the proposed algorithm outperforms the existing algorithms.
\end{abstract}

Keywords: wireless video sensor networks; non-uniform motion; K-coverage; prediction model.

\section{Introduction}

Recently wireless video sensor networks(WVSNs) have a wide range of application in areas, such as monitoring and surveillance, traffic management, and health care[1,3]. Compared with the traditional sensor networks, the biggest difference of WVSNs is the function of node, which is capable of collecting video information, processing and providing more precise image information in the meantime. WVSNs is a new frontier and comprehensive research field involved with sensor technology, embedded technology, video technology, wireless communication technology and distributed information processing technology. As a kind of wireless sensor networks, besides the advantages of common features, WVSNs has its own advantages. For example, network capacity enhancement, including node storage capacity and bandwidth resources. Rich media information, including static images, dynamic video, color, a more comprehensive resolution. Complexity of processing, including video compression coding, image feature extraction, object recognition, data fusion.

One of the most important problems in WVSNs is how to make nodes adjust their field-ofviews(FoV) to enhance the quantity and quality of information collection through network communication. The coverage problem is the mainly about monitoring the physical world in hotspots with constrained capacity of network node energy, communication bandwidth and processing[2,7,9]. The deployment of nodes and effective scheduling is the key technology of coverage problem. WVSNs coverage control theory can be divided into regional coverage and target coverage considering the point of view of coverage. It can also be divided into static target coverage and moving target coverage in the form of the target.

\footnotetext{
${ }^{\text {a }}$ Corresponding author : jyb106@zjut.edu.cn
} 
The moving target is not a simple uniform motion in real life while may under variable, but previous studies of the minimum rotation angle of the moving target K-coverage research, it has not been taken into consideration.

In this paper, we extend the K-coverage for non-uniform motion target problem, and propose a Kcoverage algorithm based on prediction for non-uniform motion target[5]. Because of the difficulty of global information acquisition, we put forward a K-coverage dynamic optimization algorithm. Beside we propose a non-uniform motion target trajectory prediction model which takes all the possible location for each moving target in the next moment into consideration. Simulation results show that the coverage quality of non-uniform motion targets has been improved apparently.

\section{Related work}

First we define the appropriate video sensor nodes perceptual model, and then analysis the work process of monitoring node. After set reasonable assumption and propose the mathematical model of coveragerate evaluation for variable motion, we establish nonlinear optimization model.

\subsection{Rotatable direction perception model}

In this paper, we set video sensor node based on directional sensor model. The sensing area is a sector on the 2-D model[2], whose center is node, sensing distance is the radius $R$ and the angle is .

Definition 1: Rotatable Direction Perception Model: Sensing range of video sensor node is a area whose center is the node, sensing distance is the radius $R$ and angle is . is the angle of the centerline in this area, and is rotational angular velocity of a node. So we can use a quintuple to describe the rotatable direction perception model, which is shown in the Figure 1 as follows:

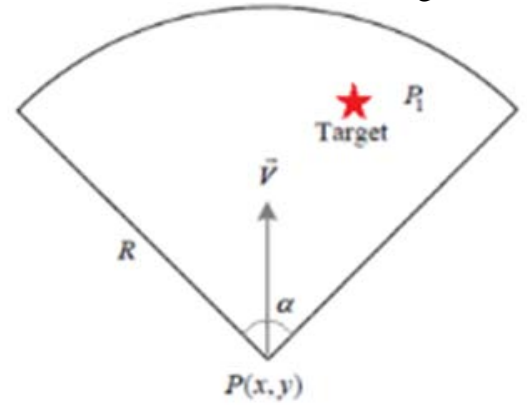

Figure 1. Rotatable Direction Perception Model

\subsection{Problem overview}

Before the discussion of non-uniform motion target coverage problem, we make following assumptions: (1) All nodes of the video sensor network are isomorphic, it means parameters of sensing node are the same. (2) Once all nodes in a video sensor network are deployed, the position will be constant. (3) Each node in video sensor network knows its own position and sensing direction angle. Meanwhile each node is able to control the sensing direction by itself. (4) Real-time position of the motion target and velocity information can be obtained by radar / acoustic sensor. (5) The target doing non-uniform motion throughout the whole process, but in short interval, it assume that acceleration is constant. (6) Moving targets do not influence each other.

Suppose in a given monitoring area, a set of target and a set of directional sensor node, each directional sensor node is represented by a quintuple, the sensing radius, FoV and rotational angular velocity are the same. Each directional sensor can make clockwise rotation or counterclockwise rotation, it is means that directional sensor node, whose initial direction angle is, could rotate 
clockwise or counterclockwise in degrees, . After the rotation, parameters of node is updated as . So new direction angle is.

\subsection{Non-uniform motion target trajectory prediction model}

Currently main prediction methods for target tracking includes particle filter, Kalman filtering etc ${ }^{[5,8]}$, but these methods require a large number of iterations and high processing power from each node. Although these techniques are more accurate, they are not suitable for video sensor nodes which have limited resources and low calculate ability.

In this paper, our model is based on probability. As the motion of target is non-uniform, at time $t+1$, its possible reach area namely $A$ will not be a regular shape. If node can rotate itself to make the sensing area cover area $A$ completely, then it will be able to cover the target at time $t+1$. Based on the current position of target, the nearby video sensor nodes are able to adjust their working direction to achieve the K-coverage of target . There are two types of nodes, one can completely cover the area Aat time $t+1$ as mentioned before, the other can partially cover the area $A$ of at time $t+1$ and it still has chance to cover in the next moment, both situations make the tracking node set .

Thus, we only need to consider the nodes of tracking node set of the tracked target , and then make decisions in the next moment. Based on suppose 5 , at time $t+1$ the most likely reach position of moving target is the location where target continue to move in with acceleration at time $t$ and reached after a interval .

To this end, for the acceleration change at time $t+1$, this paper introduces the probability density function:

Definition 2: The joint probability density function of moving target acceleration changes : In next moment, the target acceleration changes randomly, both the velocity and direction, represented by $X$, $Y$ respectively. So represents the probability density function of target acceleration change in velocity $\mathrm{x}$ and in direction $\mathrm{y}$, represents the distribution function of $x, y$, represents the probability value of that $X$ in the interval of $[a, b]$ and $Y$ in the interval of $[c, d]$. Since the velocity of the target acceleration have upper and lower limits. So , and it satisfies:

$$
\left\{\begin{array}{l}
\varphi(x, y) \geq 0 \\
P\left(a_{\min } \leq X \leq a_{\max },-\pi \leq Y \leq \pi\right)=\Phi\left(a_{\max }, \pi\right)-\Phi\left(a_{\min },-\pi\right)=\int_{-\pi}^{\pi} \int_{a_{\min }}^{a_{\max }} \varphi(x, y) d x d y=1
\end{array}\right.
$$

\subsection{Problem analysis and definition}

Combined with the above coverage probability evaluation model, at time $t+1$, the possibility of target move with acceleration could be calculated. Meanwhile the possibility of node cover target is . The function of is:

$$
g_{i j}(x, y)=\left\{\begin{array}{cc}
1 & \text { if } S_{i} \text { suspected } T_{j} \\
0 & \text { otherwise }
\end{array}\right.
$$

Therefore, at time $t$, it requires to adjust the monitoring direction of each tracking node in order to increase the probability of nodes in the network for covering moving target. The method for determining whether target is covered by video sensor node at time $t$ is and the vector direction located in .

Definition 3: Minimum Rotation Angle Of Non-Uniform Motion Objects Coverage Problem: It requires each moving target appearing in monitoring area is covered by at least $K$ different sensor nodes every moment. By rotating in a rotational angular velocity to adjust node monitoring direction, and under the precondition of multiple moving targets meet the K-coverage requirements, to minimize the sum of the tracking node rotation angle in the covering area. 
From this we can obtain the following nonlinear optimization model:

$$
\left\{\begin{aligned}
& \min \int_{0}^{T} \sum_{i=1}^{n}\left|\Delta \theta_{i, t}\right| d t \\
& \text { s.t. } {\left[0.5+\sum_{i=1}^{n} \int_{a_{\min }}^{a_{\max }} \int_{-\pi}^{\pi}\left(\Phi_{j, t}(x, y)-\Phi_{j, t}\left(a_{\min },-\pi\right)\right) g_{i j, t}(x, y) d x d y\right] \geq K } \\
& \Delta \theta_{i, t} \in[-\pi, \pi] \cap[-\omega \Delta t, \omega \Delta t] \\
& \forall i=1,2, \cdots, n \forall j=1,2, \cdots, m \quad \forall t=0,1,2, \cdots
\end{aligned}\right.
$$

The objective function represents that during the process of every moving target from entering into the monitoring area to leaving the boundary, under the precondition of target meet K-coverage requirement every moment, minimize the sum of sensor node rotation angle. Constraint condition represents at time $t$, each motion target is at least covered by $K$ different video sensor nodes, we utilize rounding calculations, and limit the maximum angle of each node rotation by the rotational angular velocity .

\section{Distributed coverage algorithm based on prediction}

The problem that we study is a NP complete problem, so it is difficult to solve the nonlinear optimization problem we mentioned in last section in polynomial time. In order to solve this problem, we need to find a new distributed optimal algorithm.

In this section, we propose a K-coverage dynamic optimization algorithm, which takes all the possible positions that the non-uniform moving target will reach each time into consideration, and exchanges information with its neighbor nodes. Then, by using the non-uniform motion target trajectory prediction model, we can make decision with the coverage probability value in turn. And each moment decision made ahead of time by rotation, which effectively avoid the delay of decisionmaking.

The core idea of the algorithm: we have fully considered all the positions the non-uniform motion target will reach next time, the irregular area $A$. And based on the target trajectory prediction model in last section, the node can calculate the coverage probability of the position itself and produce a forecast table $C$ of the coverage probability. is the possible position that target may reach under the sensing angle of the node . Table $C$ is as follow:

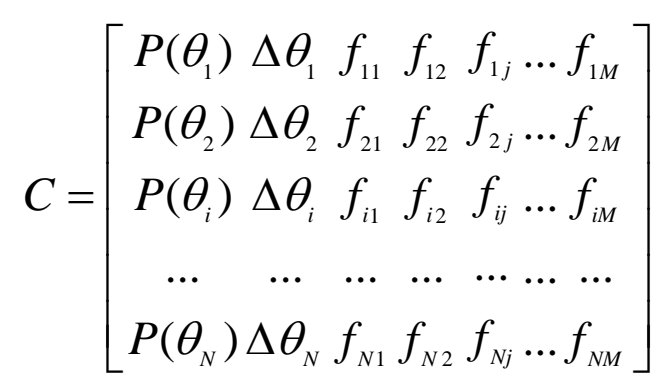

$\mathrm{M}$ is the maximum number of the moving targets that fail to reach the K-coverage that the node can be covered. Besides $N$ is the number of all the neighbor nodes of node. Each row in table $C$ records when the sensing angle of node is , the maximum value of coverage probability prediction can reaches, and the calculating formula of is:

$$
P\left(\theta_{i}\right)=\sum_{j=1}^{M} f_{i j}
$$


is the coverage probability of the position that the target may reach when the sensing angle is

Every moment, video sensor node start form the current sensing angle, calculating the deviation angle, when coverage probability prediction $P$ reach maximum within a range of , and recorded in table $C$. Then broadcast table $C$ to the neighbor nodes within communication, sort node in descending order by $P$, if agreement, select the node with maximum coverage probability to have priority, if the expected coverage values reach $K$, it can approximately deem that the target have met K-coverage requirements, rotate the angle, repeat until the target is reached $\mathrm{K}$ level coverage requirements or cover the area are selected node.

Based on above analysis, this paper have proposed K-coverage Prediction for Non-uniform Motion Objects Algorithm (KPNMOA). Starting from the first moment, based on the current target position, we can estimate the covering area. Each node makes decision by the value of coverage probability prediction $P$.

\section{Simulation result}

We will perform our simulate process and analysis results in this section. Simulation software was developed on the Microsoft .Net Framework and finished multiple experiments. Simulation environment set as follows: The size of monitoring area is $500 \times 500$, count of sensor is 150 , radius is $50, \mathrm{FoV}$ is $60^{\circ}$, direction range is $[0,2 \pi]$, maximum angular velocity is $30 \%$, MaxK is 4 , initial velocity is $10 \mathrm{~m} / \mathrm{s}$, acceleration range is $[-5,5]$, interval is $1 \mathrm{~s}$.

Besides set basic parameters as above, we optimize an evaluation function of coverage quality, which is influenced by the duration of coverage and the number of nodes coveraged. The calculating formula of coverage quality is:

$$
Q=\sum_{i=1}^{M} K_{i} t_{i}^{2}
$$

is the weight of the number of nodes coverage, is the duration of coverage.

We compare coverage quality of five kinds of algorithm includes Random, Continue, DPGKCA[4], MPKCDA[6] and KPNMOA, under the same environmental parameters.

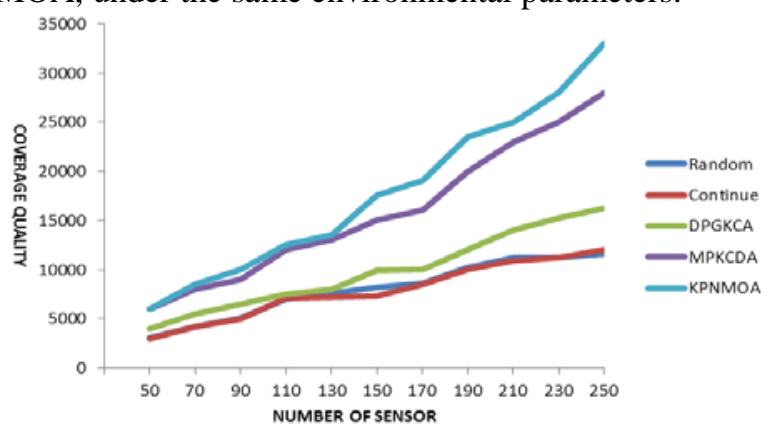

Figure 2. Impact of number of sensor on coverage quality

Figure 2 shows performances of five algorithms under different node scales. When the quantity of nodes is confirm, algorithm Random and Continue perform similarly. DPGKCA is better than Random and Continue and KPNMOA is better than MPKCDA and as the sensor nodes increases. The performance of KPNMOA increases notably. 


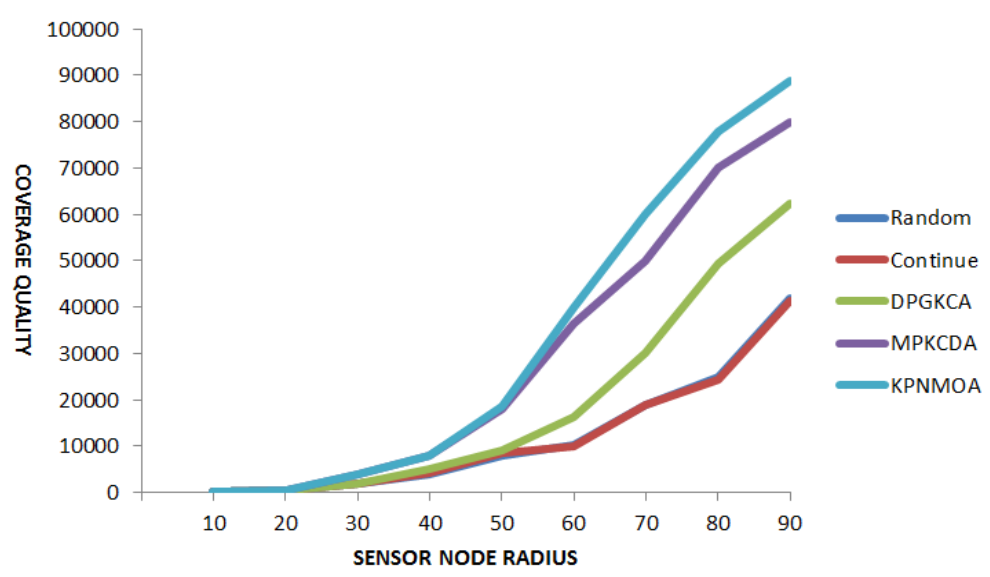

Figure 3. Impact of sensor node radius on coverage quality

Figure 3 shows the impact of sensing radius on coverage quality. With the increase of radius, coverage quality rises exponentially. In addition KPNMOA has an advantage over DPGKCA and MPKCDA obviously.

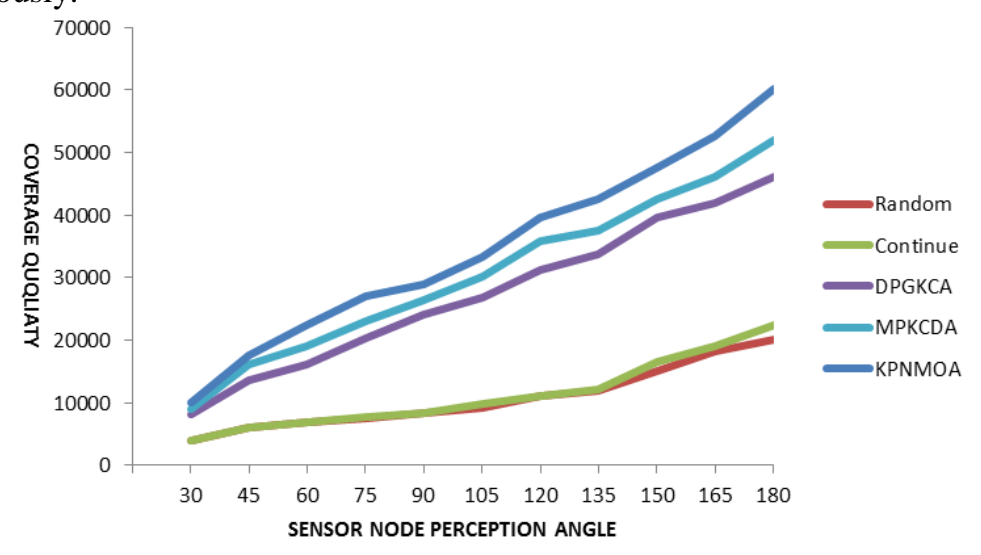

Figure 4. Impact of sensor node perception angle on coverage quality

Figure 4 shows the influence of sensing angle to coverage quality. It is obvious that with the increase of radius, KPNMOA perform better than DPGKCA and MPKCDA.

\section{Conclusion and future works}

This paper studies the K-coverage problem for non-uniform motion objects. First, we describe rotatable direction perception model of video sensor nodes. Based on the sensing model, we put forward the non-uniform motion target trajectory prediction model, which is used to guide sensor nodes to rotate. Compared with the previous, it takes the acceleration into consideration and help us to analyze the possible havior of motion object in area more accurately. Then, we propose a algorithm for optimization, which improves the quality of coverage while reducing the energy consumption of the coverage. Finally, the simulation results show the effectiveness of the algorithm. In later work, we consider to add the obstacles[3], which will help us to simulate the real environment coverage even better. 


\section{Acknowledgments}

This work is supported by the National Natural Science Foundation of China No.61379123, No.61402415 and the National Key Technology R\&D Program during the Twelfth Five-year Plan Period No.2012BAD10B0101.

\section{References}

1. Vikram P. Munishwar, Nael B. Abu-Ghazaleh, Coverage Algorithms for Visual Sensor Networks ACM, Transactions on Sensor Networks 9, 45 (2013)

2. D Tao, HD Ma, Coverage Control Algorithms for Directional Sensor Networks, Journal of Software 22, 10 (2011)

3. YB Jiang, WL Wang, WJ Chen, Coverage Optimization of Occlusion-Free Surveillance for Video Sensor Networks, Journal of Software 23,10 (2012)

4. YB Jiang, Q Chen, WL Wang, H Lou, A K level Coverage Enhancement Algorithm Based on Moving Target Trajectory Prediction for Video Sensor Networks, Chinese Journal of Sensors and Actuators, 27 (2014)

5. J Ren, Q Xiong, W Shi, A Target Tracking Algorithm Based on Prediction, Chinese Journal of Sensors And Actuators 24, 10 (2011)

6. YB Jiang, Q Chen, WL Wang, H Lou, JW Zheng, Multipath K level coverage dynamic optimization algorithm for video sensor networks, Chinese Journal of Scientific Instrument, 36 (2015)

7. M. Amac Guvensan, A. Gokhan Yavuz, On coverage issues in directional sensor networks: A survey, Ad Hoc Networks, 9 (2011)

8. K Shi, H Chen, Y Lin, Probabilistic coverage based sensor scheduling for target tracking sensor networks, Information Sciences 292, 19 (2015)

9. Md. Mofijul Islam, Md. Ahasanuzzaman, Md. Abdur Razzaque, Mohammad Mehedi Hassa, Abdulhameed Alelaiwi, Yang Xiang, Target coverage through distributed clustering in directional sensor networks, Eurasip Journal on Wireless Communications and Networking 2015, 1 (2015) 